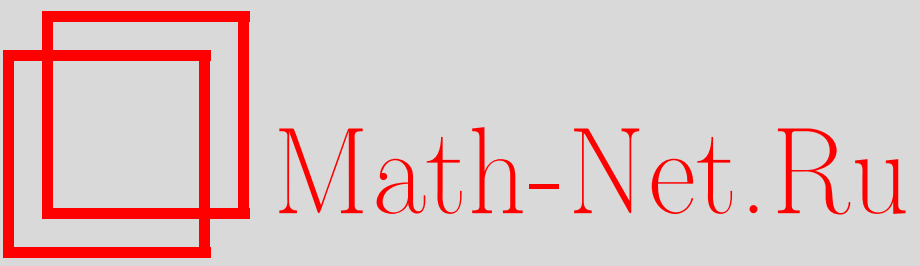

А. Ю. Бабанский, Ю. А. Ситенко, Об индуцировании вакуумной энергии сингулярным магнитным вихрем, ТМФ, 1999, том 120, номер 1, 72-81

DOI: https://doi.org/10.4213/tmf761

Использование Общероссийского математического портала Math-Net.Ru подразумевает, что вы прочитали и согласны с пользовательским соглашением

http://www.mathnet.ru/rus/agreement

Параметры загрузки:

IP : 54.224 .135 .184

26 апреля 2023 г., 14:03:17 
ТЕОРЕТИЧЕСКАЯ

И МАТЕМАТИЧЕСКАЯ

ФИЗИКА

Том 120, № 1

июль, 1999

(C) 1999 г. $\quad$ А. Ю. Бабанский*, Ю. А. Ситенко*

\section{ОБ ИНДУЦИРОВАНИИ ВАКУУМНОЙ ЭНЕРГИИ СИНГУЛЯРНЫМ МАГНИТНЫМ ВИХРЕМ}

Конфигурация внешнего статического магнитного поля в виде сингулярного вихря поляризует вакуум квантованного заряженного скалярного поля в пространстве произвольной размерности. С использованием двух методов - дзета-функции и ядра теплопроводности - решена проблема регуляризации и устранения ультрафиолетовой расходимости и определена плотность индуцированной вакуумной энергии. Сформулировано достаточное условие применимости метода дзета-функции к задачам в пространствах произвольной размерности.

\section{1. ВВЕДЕНИЕ}

Вопрос индуцирования вакуумной энергии внешними полями имеет достаточно длительную историю и освешается во многих монографиях (см., например, [1-3]). В настоящей работе ставится задача определения вакуумной энергии, индуцированной конфигурацией внешнего статического магнитного поля в виде сингулярного вихря (см. также [4]). Нами показано, что эта задача имеет решение в случае пространств произвольной размерности $d \geqslant 2$.

Оператор вторично квантованного комплексного скалярного поля представляется в виде

$$
\psi\left(x^{0}, \mathbf{x}\right)=\sum_{\lambda} \frac{1}{\sqrt{2 E_{\lambda}}}\left(e^{-i E_{\lambda} x^{0}}\langle\mathbf{x} \mid \lambda\rangle a_{\lambda}+e^{i E_{\lambda} x^{0}}\langle\mathbf{x} \mid-\lambda\rangle b_{\lambda}^{+}\right)
$$

где $a_{\lambda}^{+}$и $a_{\lambda}\left(b_{\lambda}^{+}\right.$и $\left.b_{\lambda}\right)$ - операторы рождения и уничтожения скалярных частиц (античастиц), удовлетворяюшие коммутационным соотношениям

$$
\left[a_{\lambda}, a_{\lambda^{\prime}}^{+}\right]_{-}=\left[b_{\lambda}, b_{\lambda^{\prime}}^{+}\right]_{-}=\left\langle\lambda \mid \lambda^{\prime}\right\rangle
$$

$\langle\mathbf{x} \mid \lambda\rangle$ - решение стационарного уравнения Клейна-Гордона

$$
\left(-\nabla^{2}+m^{2}\right)\langle\mathbf{x} \mid \lambda\rangle=E_{\lambda}^{2}\langle\mathbf{x} \mid \lambda\rangle
$$

\footnotetext{
* Институт теоретической физики им. Н. Н. Боголюбова НАН Украины, Киев, Украина
} 
$\boldsymbol{\nabla}$ - оператор ковариантного дифференцирования во внешнем (фоновом) поле, $\lambda$ - набор параметров (квантовых чисел), от которых зависит состояние, $E_{\lambda}=E_{-\lambda}>0$ - энергия состояния; в (1) подразумевается суммирование по дискретным и интегрирование (с определенной мерой) по непрерывным значениям $\lambda$.

Стандартным образом можно получить формальное выражение для плотности вакуумной энергии

$$
\varepsilon(\mathbf{x})=\left\langle\mathbf{x}\left|\left(-\nabla^{2}+m^{2}\right)^{\frac{1}{2}}\right| \mathbf{x}\right\rangle
$$

Это выражение плохо определено, поскольку соответствующий ему интеграл расходится в импульсном пространстве как $p^{d+1}$ при $p \rightarrow \infty$. Для регуляризации этой (ультрафиолетовой $(У \Phi))$ расходимости добавим под знак интеграла экспоненциально убывающий при $p \rightarrow \infty$ множитель и определим регуляризованную плотность вакуумной энергии

$$
\varkappa_{\mathbf{x}}(t)=\left\langle\mathbf{x}\left|\left(-\nabla^{2}+m^{2}\right)^{\frac{1}{2}} \exp \left[-t\left(-\nabla^{2}+m^{2}\right)\right]\right| \mathbf{x}\right\rangle
$$

где $t>0$. Нетрудно видеть, что выражение $(5)$ расходится при $t \rightarrow 0_{+}$даже в свободной теории, т.е. в отсутствие какого-либо взаимодействия квантованного скалярного поля. В свободной теории от вакуумных расходимостей избавляются с помошью требования нормального упорядочения произведения операторов (см., например, [1-3]). Поэтому физический интерес может представлять не сама величина $\varkappa_{\mathbf{x}}(t)$, а разность

$$
\varkappa_{\mathbf{x}}^{\text {ren }}(t)=\varkappa_{\mathbf{x}}(t)-\varkappa_{\mathbf{x}}^{(0)}(t)
$$

где $\varkappa_{\mathbf{x}}^{(0)}(t)$ - регуляризованная плотность вакуумной энергии в свободной теории, которая получается при замене в выражении (5) ковариантной производной на обычную: $\nabla \rightarrow \partial$

Обладает ли величина $\varkappa_{\mathbf{x}}^{\text {ren }}(t)(6)$ конечным пределом при $t \rightarrow 0_{+}$? В общем случае ответ на этот вопрос отрицателен, поскольку для получения конечного результата наряду с выгитанием (6) могут потребоваться также и бесконечная в пределе $t=0_{+}$перенормировка констант взаимодействия с внешними полями, и добавление в лагранжиан бесконечных в пределе $t=0_{+}$контрчленов. В частности, именно такая ситуация реализуется в случае индуцирования вакуумной энергии однородным электромагнитным полем [1] или кривизной пространства [3]. Более того, как подчеркивается в работе [5], ситуация изменяется кардинальным образом при изменении размерности пространства $d$ : в случае $d=3$ требуется перенормировка заряда (т.е. добавление контрчлена в виде инварианта второй степени по напряженности внешнего поля), в то время как в случаях $d=2$ и $d=4$ - не требуется, а при $d \geqslant 5$ одной перенормировки заряда недостаточно и необходимо добавление контрчленов в виде инвариантов третьей и выше степеней по напряженности внешнего поля. Однако в настояшей работе показано, что в случае индуцирования вакуумной энергии сингулярным магнитным вихрем предел величины $\varkappa_{\mathbf{x}}^{\mathrm{ren}}(t)(6)$ при $t \rightarrow 0_{+}$сушествует при любом значении $d \geqslant 2$.

Итак, в качестве внешнего выбирается статическое магнитное поле, и ковариантная производная принимает вид

$$
\nabla=\boldsymbol{\partial}-i \mathbf{V}(\mathbf{x})
$$


где $\mathbf{V}(\mathbf{x})$ - векторный потенциал поля. Отметим, что в $d$-мерном пространстве напряженность магнитного поля является антисимметричным тензором ранга $d-2$ :

$$
B^{\nu_{1} \ldots \nu_{d-2}}(\mathbf{x})=\left[\partial_{\mu_{1}} V_{\mu_{2}}(\mathbf{x})\right] \epsilon^{\mu_{1} \mu_{2} \nu_{1} \ldots \nu_{d-2}},
$$

$\epsilon^{\mu_{1} \ldots \mu_{d}}-$ полностью антисимметричный тензор, $\epsilon^{12 \ldots d}=1$. Конфигурация магнитного поля выбирается в виде сингулярного вихря (точка в случае $d=2$, линия в случае $d=3$, $(d-2)$-мерная гиперповерхность в случае $d>3)$ :

$$
\begin{aligned}
V_{1}(\mathbf{x})=-\Phi \frac{x^{2}}{\left(x^{1}\right)^{2}+\left(x^{2}\right)^{2}}, \quad V_{2}(\mathbf{x}) & =\Phi \frac{x^{1}}{\left(x^{1}\right)^{2}+\left(x^{2}\right)^{2}}, \quad V_{\nu}(\mathbf{x})=0, \quad \nu=\overline{3, d}, \\
B^{3 \ldots d}(\mathbf{x}) & =2 \pi \Phi \delta\left(x^{1}\right) \delta\left(x^{2}\right),
\end{aligned}
$$

$\Phi$ - поток вихря (в единицах $2 \pi$ ). Регулярное решение уравнения Клейна-Гордона (3) в поле вихря (9) с учетом (7) имеет вид

$$
\langle\mathbf{x} \mid k n \mathbf{p}\rangle=(2 \pi)^{\frac{1-d}{2}} J_{|n-\Phi|}(k r) e^{i n \varphi} e^{i \mathbf{p} \mathbf{x}_{d-2}}
$$

где

$$
0<k<\infty, \quad n \in \mathbb{Z}, \quad-\infty<p_{\nu}<\infty, \quad \nu=\overline{3, d}
$$

$J_{\mu}(z)$ - функция Бесселя порядка $\mu, r=\sqrt{\left(x^{1}\right)^{2}+\left(x^{2}\right)^{2}}, \varphi=\operatorname{arctg}\left(x^{2} / x^{1}\right), \quad \mathbf{x}_{d-2}=$ $\left(0,0, x^{3}, \ldots, x^{d}\right), \mathbb{Z}$ - множество целых чисел.

Как известно, в задаче об эффекте Казимира [6] для определения перенормированной вакуумной энергии достаточно лишь одного вычитания и нет необходимости в добавлении контрчленов (см., например, [2]). Поскольку согласно (10) напряженность внешнего магнитного поля обрашается в нуль везде вне гиперповерхности $x^{1}=x^{2}=0$, а известные до сих пор в задачах индуцирования вакуумной энергии внешними условиями контрчлены являются инвариантами, построенными из напряженности (из кривизн слоя и базы расслоенных многообразий в общем случае), то можно ожидать, что и в задаче, рассматриваемой в настояшей работе, достаточно лишш одного вычитания. Эти ожидания оправдываются, и, как будет показано ниже, действительно, никакие аномальные контрчлены не возникают.

Однако одной только конечности (согласованной с нормальным упорядочением произведения операторов в свободной теории) не достаточно для того, чтобы результату можно было придать физический смысл. Другим условием является независимость результата от способа регуляризации УФ-расходимости - ведь именно в однозначности определения конечного результата и состоит решение проблемы УФ-расходимости. В связи с этим рассмотрим функцию

$$
\sigma_{\mathbf{x}}(s, t)=\left\langle\mathbf{x}\left|\left(-\nabla^{2}+m^{2}\right)^{-s} \exp \left[-t\left(-\nabla^{2}+m^{2}\right)\right]\right| \mathbf{x}\right\rangle
$$


(где $s$ - комплексный параметр), которая объединяет возможности экспоненциальной и степенной регуляризаций. Если в (13) перейти к пределу $t=0_{+}$, то соответствуюший интеграл оказывается сходяшимся лишь при $\operatorname{Re} s>d / 2$. Таким образом, использование степенной регуляризации (так называемый метод дзета-функции) с необходимостью предполагает некоторую процедуру аналитического продолжения, для того чтобы перейти к представляющим интерес значениям $s(s=-1 / 2$ в случае вакуумной энергии). Метод дзета-функции широко применяется к задачам индуцирования вакуумной энергии, в частности к задачам об эффекте Казимира и его обобшениях (см., например, [7]). Однако, как показано в работе [5], в случае пространств высшей размерности этот метод может приводить к результатам, не имеющим физического смысла, и поэтому использование экспоненциальной регуляризации (метод ядра теплопроводности) является предпочтительным. Нами будет показано, что к задаче индуцирования вакуумной энергии сингулярным магнитным вихрем в пространстве любой размерности $d \geqslant 2$ метод дзета-функции действительно применим и что оба указанных метода приводят к одному результату. Тем самым будет решена проблема регуляризации и устранения УФ-расходимости и однозначно определена конечная перенормированная плотность вакуумной энергии, имеющая физический смысл.

Отметим, что наряду с очевидным соотношением

$$
\sigma_{\mathbf{x}}\left(-\frac{1}{2}, t\right)=\varkappa_{\mathbf{x}}(t)
$$

мы будем пользоваться следующим выражением:

$$
\sigma_{\mathbf{x}}(0, t)=\left\langle\mathbf{x}\left|\exp \left[-t\left(-\nabla^{2}+m^{2}\right)\right]\right| \mathbf{x}\right\rangle,
$$

определяющим ядро теплопроводности, в терминах которого и будет сформулировано достаточное условие применимости метода дзета-функции.

Используя явный вид решений уравнения Клейна-Гордона (11), получим

$$
\begin{aligned}
\sigma_{\mathbf{x}}(s, t)= & (2 \pi)^{1-d} \int d^{d-2} p \int_{0}^{\infty} d k k\left(p^{2}+k^{2}+m^{2}\right)^{-s} \times \\
& \times \exp \left[-t\left(p^{2}+k^{2}+m^{2}\right)\right] \sum_{n \in \mathbb{Z}} J_{|n-\Phi|}^{2}(k r) .
\end{aligned}
$$

Нами будет показано, что выражение (16) имеет степенную расходимость $t^{s-d / 2}$ при $t \rightarrow 0_{+}$в случае $\operatorname{Re} s<d / 2$, в то время как перенормированная функция

$$
\sigma_{\mathbf{x}}^{\text {ren }}(s, t)=\sigma_{\mathbf{x}}(s, t)-\sigma_{\mathbf{x}}^{(0)}(s, t)
$$

$\left(\right.$ где $\sigma_{\mathbf{x}}^{(0)}(s, t)$ получается из $(16)$ при $\Phi=0$ ) разлагается в ряд Тейлора по степеням $t$ :

$$
\sigma_{\mathbf{x}}^{\mathrm{ren}}(s, t) \underset{t \rightarrow 0_{+}}{\backsim} \sum_{n=0}^{\infty} \chi_{\mathbf{x}}(s-n) \frac{(-t)^{n}}{n !},
$$

где $\chi_{\mathbf{x}}(s)$ - голоморфная функция на всей комплексной плоскости $s$, имеющая нули при целых неположительных вешественных значениях $s$. Тем самым будет доказано сушествование перенормированной плотности вакуумной энергии

$$
\varepsilon^{\mathrm{ren}}(\mathbf{x})=\lim _{t \rightarrow 0_{+}} \sigma_{\mathbf{x}}^{\mathrm{ren}}\left(-\frac{1}{2}, t\right)=\chi_{\mathbf{x}}\left(-\frac{1}{2}\right) .
$$




\section{2. ОПРЕДЕЛЕНИЕ ФУНКЦИИ $\sigma_{\mathbf{x}}(s, t)$}

Выполняя интегрирование по угловой части векторной переменной ри суммирование по $n$, представим выражение (16) в виде суммы двух слагаемых, из которых первое не зависит от расстояния до вихря:

$$
\sigma_{\mathbf{x}}^{(0)}(s, t)=\frac{4}{(4 \pi)^{\frac{d}{2}} \Gamma\left(\frac{d}{2}-1\right)} \int_{0}^{\infty} d p p^{d-3} \int_{0}^{\infty} d k k \frac{\exp \left[-t\left(p^{2}+k^{2}+m^{2}\right)\right]}{\left(p^{2}+k^{2}+m^{2}\right)^{s}},
$$

а второе, как будет показано ниже, экспоненциально убывает на больших расстояниях от вихря:

$$
\begin{aligned}
\sigma_{\mathbf{x}}^{\mathrm{ren}}(s, t)= & -\frac{4}{(4 \pi)^{\frac{d}{2}} \Gamma\left(\frac{d}{2}-1\right)} \int_{0}^{\infty} d p p^{d-3} \int_{0}^{\infty} d k k \frac{\exp \left[-t\left(p^{2}+k^{2}+m^{2}\right)\right]}{\left(p^{2}+k^{2}+m^{2}\right)^{s}} \times \\
& \times \int_{k r}^{\infty} d y\left[J_{F}(y) J_{-1+F}(y)+J_{1-F}(y) J_{-F}(y)\right]
\end{aligned}
$$

здесь $Г(z)$ - гамма-функция Эйлера и

$$
F=\Phi-[[\Phi]], \quad 0 \leqslant F<1,
$$

$[[u]]$ обозначает целую часть величины $u$ (ближайшее к $u$ и не превьшающее $u$ целое число). Отметим, что из выражения (16) следует периодичность результатов по величине потока вихря $\Phi$ (т.е. зависимость только от величины $F(22)$ ), а также симметрия относительно замены $F \rightarrow 1-F$.

Выполняя интегрирование по $p$ и $k$ в выражении (20), имеем

$$
\sigma_{\mathbf{x}}^{(0)}(s, t)=\frac{e^{-t m^{2}} m^{d-2 s}}{(4 \pi)^{\frac{d}{2}}} \Psi\left(\frac{d}{2}, \frac{d}{2}-s+1 ; t m^{2}\right),
$$

где

$$
\Psi(a, b ; z)=\frac{1}{\Gamma(a)} \int_{0}^{\infty} d y y^{a-1}(y+1)^{b-a-1} e^{-z y}
$$

- вырожденная гипергеометрическая функция Трикоми (см., например, [8]). Учитывая ее асимптотику при $z \rightarrow 0$, получим

$$
\begin{array}{ll}
\sigma_{\mathbf{x}}^{(0)}(s, t) \underset{t \rightarrow 0_{+}}{\simeq} \frac{m^{d-2 s}}{(4 \pi)^{\frac{d}{2}}} \frac{\Gamma\left(s-\frac{d}{2}\right)}{\Gamma(s)}+\cdots, & \operatorname{Re} s>\frac{d}{2}, \\
\sigma_{\mathbf{x}}^{(0)}(s, t) \underset{t \rightarrow 0_{+}}{\simeq} \frac{\ln \left(t m^{2}\right)^{-1}-2 \gamma-\psi\left(\frac{d}{2}\right)}{(4 \pi)^{\frac{d}{2}} \Gamma\left(\frac{d}{2}\right)}+\cdots, & s=\frac{d}{2}, \\
\sigma_{\mathbf{x}}^{(0)}(s, t) \underset{t \rightarrow 0_{+}}{\simeq} \frac{t^{s-\frac{d}{2}}}{(4 \pi)^{\frac{d}{2}}} \frac{\Gamma\left(\frac{d}{2}-s\right)}{\Gamma\left(\frac{d}{2}\right)}+\cdots, & \operatorname{Re} s<\frac{d}{2},
\end{array}
$$

где $\psi(z)=\frac{d}{d z} \ln \Gamma(z)-$ дигамма-функция и $\gamma=-\psi(1)-$ постоянная Эйлера. 
Рассмотрим выражение (21). Используя интегральное представление

$$
\begin{aligned}
\left(p^{2}+k^{2}+m^{2}\right)^{-s}= & \frac{(-1)^{N}}{\Gamma(s+N)} \int_{t}^{\infty} d w(w-t)^{s+N-1} \times \\
& \times \frac{\partial^{N}}{\partial w^{N}} \exp \left[-(w-t)\left(p^{2}+k^{2}+m^{2}\right)\right], \\
\operatorname{Re} s> & -N, \quad N=0,1,2, \ldots,
\end{aligned}
$$

и выполняя интегрирование по $p$, находим

$$
\begin{aligned}
& \sigma_{\mathbf{x}}^{\text {ren }}(s, t)=-\frac{2}{(4 \pi)^{\frac{d}{2}}} \frac{(-1)^{N}}{\Gamma(s+N)} \int_{t}^{\infty} d w(w-t)^{s+N-1} \times \\
& \quad \times \frac{\partial^{N}}{\partial w^{N}}\left\{\frac{e^{-w m^{2}}}{w^{\frac{d}{2}-1}} \int_{0}^{\infty} d k k e^{-w k^{2}} \int_{k r}^{\infty} d y\left[J_{F}(y) J_{-1+F}(y)+J_{1-F}(y) J_{-F}(y)\right]\right\} .
\end{aligned}
$$

Учитывая соотношение

$$
\begin{gathered}
\int_{0}^{\infty} d k k e^{-w k^{2}} \int_{k r}^{\infty} d y\left[J_{F}(y) J_{-1+F}(y)+J_{1-F}(y) J_{-F}(y)\right]= \\
=\frac{\sin (F \pi)}{2 \pi w} \int_{\frac{r^{2}}{2 w}}^{\infty} d u e^{-u}\left[K_{F}(u)+K_{1-F}(u)\right]
\end{gathered}
$$

где $K_{\mu}(z)$ - функция Макдональда порядка $\mu$, получаем

$$
\begin{aligned}
\sigma_{\mathbf{x}}^{\mathrm{ren}}(s, t)= & -\frac{4 \sin (F \pi)}{(4 \pi)^{\frac{d}{2}+1}} \frac{(-1)^{N}}{\Gamma(s+N)} \int_{t}^{\infty} d w(w-t)^{s+N-1} \times \\
& \times \frac{d^{N}}{d w^{N}}\left\{\frac{e^{-w m^{2}}}{w^{\frac{d}{2}}} \int_{\frac{r}{2 w}}^{\infty} d u e^{-u}\left[K_{F}(u)+K_{1-F}(u)\right]\right\} .
\end{aligned}
$$

Нетрудно убедиться в справедливости соотношения

$$
\sigma_{\mathbf{x}}^{\mathrm{ren}}(s, t)=-\frac{d}{d t} \sigma_{\mathbf{x}}^{\mathrm{ren}}(s+1, t) .
$$

Переходя к пределу $t=0_{+}$в $(31)$, получаем

$$
\begin{aligned}
\chi_{\mathbf{x}}(s) \equiv & \lim _{t \rightarrow 0_{+}} \sigma_{\mathbf{x}}^{\mathrm{ren}}(s, t)=-\frac{4 \sin (F \pi)}{(4 \pi)^{\frac{d}{2}+1}} \frac{(-1)^{N}}{\Gamma(s+N)} \int_{0}^{\infty} d w w^{s+N-1} \times \\
& \times \frac{d^{N}}{d w^{N}}\left\{\frac{e^{-w m^{2}}}{w^{\frac{d}{2}}} \int_{\frac{r^{2}}{2 w}}^{\infty} d u e^{-u}\left[K_{F}(u)+K_{1-F}(u)\right]\right\} .
\end{aligned}
$$

Интегрированием по частям можно привести последнее выражение к виду

$$
\chi_{\mathbf{x}}(s)=-\frac{4 \sin (F \pi)}{(4 \pi)^{\frac{d}{2}+1} \Gamma(s)} \int_{0}^{\infty} d w w^{s-\frac{d}{2}-1} e^{-w m^{2}} \int_{\frac{r^{2}}{2 w}}^{\infty} d u e^{-u}\left[K_{F}(u)+K_{1-F}(u)\right]
$$


откуда, используя интегральное представление

$$
e^{-u}\left[K_{F}(u)+K_{1-F}(u)\right]=4 \int_{1}^{\infty} d v \frac{v}{\sqrt{v^{2}-1}} \operatorname{ch}[(2 F-1) \operatorname{Arch} v] e^{-2 u v^{2}}
$$

окончательно находим

$$
\begin{aligned}
\chi_{\mathbf{x}}(s)= & -\frac{16 \sin (F \pi)}{(4 \pi)^{\frac{d}{2}+1} \Gamma(s)}\left(\frac{m}{r}\right)^{\frac{d}{2}-s} \times \\
& \times \int_{1}^{\infty} \frac{d v}{\sqrt{v^{2}-1}} \operatorname{ch}[(2 F-1) \operatorname{Arch} v] v^{s-\frac{d}{2}-1} K_{s-\frac{d}{2}}(2 m r v) .
\end{aligned}
$$

Таким образом, мы получили соотношения $(31),(32)$ и (36) в области $\operatorname{Re} s>-N$ (см. (28)), причем соотношения (32) и (36) не зависят от величины $N$. Выбирая значение $N$ как угодно большим, получаем соотношения (32) и (36) фактически на всей комплексной плоскости $s$. Тем самым доказывается справедливость разложения (18), где функция $\chi_{\mathbf{x}}(s)$ определяется выражением (36). На больших расстояниях от вихря имеем

$$
\begin{aligned}
\sigma_{\mathbf{x}}^{\text {ren }}(s, t)= & -\frac{\sin (F \pi)}{(4 \pi)^{\frac{d}{2}} \Gamma(s)} \frac{e^{-2 m r}}{m r}\left(\frac{m}{r}\right)^{\frac{d}{2}-s}\left(1-\frac{m}{r} t\right)^{s-1} \times \\
& \times\left\{1+O\left[(m r)^{-1}\right]\right\}, \quad m r \gg 1 .
\end{aligned}
$$

Отметим также, что функция $\chi_{\mathbf{x}}(s)(36)$ и, следовательно, функция $\sigma_{\mathbf{x}}^{\text {ren }}(s, t)(18)$ могут быть аналитически продолжены по переменной $d$ как голоморфные функции на всю комплексную плоскость $d$.

\section{3. ЯДРО ТЕПЛОПРОВОДНОСТИ}

Как видно из выражения $(36)$, функция $\chi_{\mathbf{x}}(s)$ голоморфна на всей комплексной плоскости $s$ и имеет простые нули при целых неположительных вешественных значениях $s$. Следовательно,

$$
\sigma_{\mathbf{x}}^{\mathrm{ren}}(0, t) \underset{t \rightarrow 0_{+}}{\simeq} 0
$$

т.е. и функция $\sigma_{\mathbf{x}}^{\text {ren }}(0, t)$, и все ее производные обрашаются в нуль в точке $t=0_{+}$. Действительно, можно выписать точное выражение

$$
\sigma_{\mathbf{x}}^{\mathrm{ren}}(0, t)=-\frac{4 \sin (F \pi)}{(4 \pi)^{\frac{d}{2}+1}} t^{-\frac{d}{2}} e^{-t m^{2}} \int_{\frac{r^{2}}{2 t}}^{\infty} d u e^{-u}\left[K_{F}(u)+K_{1-F}(u)\right]
$$

из которого в свою очередь получаем асимптотику

$$
\begin{gathered}
\sigma_{\mathbf{x}}^{\mathrm{ren}}(0, t)=-\frac{2 \sin (F \pi)}{(4 \pi)^{\frac{d+1}{2}}} t^{\frac{1-d}{2}} r^{-1} \exp \left[-t m^{2}-t^{-1} r^{2}\right]\left[1+O\left(t r^{-2}\right)\right], \\
0<t \ll r^{2}
\end{gathered}
$$


Таким образом, учитывая, что в отсутствие вихря $(F=0)$

$$
\sigma_{\mathbf{x}}^{(0)}(0, t)=\frac{e^{-t m^{2}}}{(4 \pi t)^{\frac{d}{2}}}
$$

(см. (23)), приходим к следующему асимптотическому выражению для ядра теплопроводности в присутствии вихря $(0<F<1)$ :

$$
\sigma_{\mathbf{x}}(0, t)=\frac{e^{-t m^{2}}}{(4 \pi t)^{\frac{d}{2}}}\left\{1-\sin (F \pi) \sqrt{\frac{t}{\pi}} r^{-1} e^{-t^{-1} r^{2}}\left[1+O\left(t r^{-2}\right)\right]\right\}, \quad 0<t \ll r^{2}
$$

\section{4. ПЛОТНОСТЬ ВАКУУМНОЙ ЭНЕРГИИ}

Учитывая (14), (17), (18), (23) и (36), получаем следующее представление для регуляризованной плотности вакуумной энергии (5):

$$
\varkappa_{\mathbf{x}}(t)=\frac{e^{-t m^{2}} m^{d+1}}{(4 \pi)^{\frac{d}{2}}} \Psi\left(\frac{d}{2}, \frac{d+3}{2} ; t m^{2}\right)+\sum_{n=0}^{\infty} \chi_{\mathbf{x}}\left(-\frac{1}{2}-n\right) \frac{(-t)^{n}}{n !}, \frac{r^{2}}{t} \gg 1
$$

где

$$
\begin{aligned}
& \chi_{\mathbf{x}}\left(-\frac{1}{2}-n\right)=\frac{64 \sin (F \pi)}{(4 \pi)^{\frac{d}{2}+2}}(-1)^{n} \Gamma\left(\frac{3}{2}+n\right)\left(\frac{m}{r}\right)^{\frac{d+1}{2}+n} \int_{1}^{\infty} \frac{d v}{\sqrt{v^{2}-1}} \times \\
& \times \operatorname{ch}[(2 F-1) \operatorname{Arch} v] v^{-\frac{d+1}{2}-n-1} K_{\frac{d+1}{2}+n}(2 m r v) \text {. }
\end{aligned}
$$

При $t \rightarrow 0_{+}$выражение (43) имеет ведущую расходимость вида $t^{-(d+1) / 2}$ и следущие за ней расходимости вида $t^{-(d+1) / 2+l}$, где $0<l<[[(d+1) / 2]]$, причем в случае нечетных значений $d$ имеется также логарифмическая расходимость $\ln \left(\mathrm{tm}^{2}\right)^{-1}$ (см. (25)-(27)). Однако все расходимости сокрашаются после вычитания (6):

$$
\varkappa_{\mathbf{x}}^{\text {ren }}(t) \underset{t \rightarrow 0_{+}}{\simeq} \sum_{n=0}^{\infty} \chi_{\mathbf{x}}\left(-\frac{1}{2}-n\right) \frac{(-t)^{n}}{n !} .
$$

Таким образом, для перенормированной плотности вакуумной энергии получаем выражение (см. (19) и (44))

$$
\begin{aligned}
\varepsilon^{\mathrm{ren}}(\mathbf{x}) \equiv & \lim _{t \rightarrow 0_{+}} \varkappa_{\mathbf{x}}^{\mathrm{ren}}(t)=\frac{16 \sin (F \pi)}{(4 \pi)^{\frac{d+3}{2}}}\left(\frac{m}{r}\right)^{\frac{d+1}{2}} \int_{1}^{\infty} \frac{d v}{\sqrt{v^{2}-1}} \times \\
& \times \operatorname{ch}[(2 F-1) \operatorname{Arch} v] v^{-\frac{d+3}{2}} K_{\frac{d+1}{2}}(2 m r v) .
\end{aligned}
$$




\section{5. ЗАКЛЮЧЕНИЕ}

В настоящей работе показано, что асимптотически при $t \rightarrow 0_{+}$ядро теплопроводности в присутствии сингулярного магнитного вихря отличается от ядра теплопроводности в свободной теории на величину, которая меньше любой, как угодно большой степени $t$ (см. (41) и (42)). В то же время разность регуляризованных плотностей вакуумной энергии в присутствии и в отсутствие сингулярного магнитного вихря (6) представляется в виде разложения Тейлора по степеням $t(45)$, все коэффициенты которого отличаются от нуля при нецелых значениях потока вихря $(0<F<1)$ (см. (44)). Переходом к предельному значению $t=0_{+}$определяется перенормированная плотность вакуумной энергии (46).

Отметим, что функция $\chi_{\mathbf{x}}(s)(36)$, через которую выражаются коэффициенты разложения функции $\sigma_{\mathbf{x}}^{\text {ren }}(s, t)(18)$ в ряд Тейлора по степеням $t$, совпадает с перенормированной плотностью дзета-фонкции, полученной в работе [4],

$$
\zeta_{\mathbf{x}}^{\text {ren }}(s)=\left\langle\mathbf{x}\left|\left(-\nabla^{2}+m^{2}\right)^{-s}\right| \mathbf{x}\right\rangle-\left\langle\mathbf{x}\left|\left(-\boldsymbol{\partial}^{2}+m^{2}\right)^{-s}\right| \mathbf{x}\right\rangle
$$

Последняя функция определена в области $\operatorname{Re} s>d / 2$ и затем аналитически продолжена на всю комплексную плоскость $s$. В отличие от этого в настоящей работе метод аналитического продолжения не используется, и функция $\chi_{\mathbf{x}}(s)$ определяется непосредственно на всей комплексной плоскости $s$. Совпадение функций $\chi_{\mathbf{x}}(s)$ и $\zeta_{\mathbf{x}}^{\mathrm{ren}}(s)$ обусловлено отмеченным выше фактическим совпадением при $t \rightarrow 0_{+}$ядер теплопроводности в присутствии и в отсутствие вихря. С этим, в частности, связано и то, что два разных способа регуляризации УФ-расходимости - с помощью экспоненциального множителя $\exp \left(-t E_{\lambda}^{2}\right)$ (используемый в настоящей работе) и с помощью степенного множителя $E_{\lambda}^{-1-2 s}$ (использованный в работе [4]) - приводят к одному и тому же результату для перенормированной плотности вакуумной энергии (46); подчеркнем еще раз, что способ [4] предполагает аналитическое продолжение по комплексной переменной $s$.

Как показано в работе [5], при индуцировании плотности вакуумной энергии регулярной конфигурацией напряженности внешнего статического магнитного поля разные способы регуляризации УФ-расходимости приводят к одному результату лишь в случае $d=2$, в то время как в случае $d \geqslant 3$ результаты различаются. Обусловлено это различием коэффициентов асимптотического при $t \rightarrow 0_{+}$разложения ядер теплопроводности в присутствии и в отсутствие внешнего поля. Было показано, что использование регуляризации УФ-расходимости с помощью экспоненциального множителя позволяет получить достаточно последовательным и самосогласованным образом перенормированную плотность вакуумной энергии, имеющую физический смысл. Тем самым поставлена под вопрос возможность использования в случае $d \geqslant 3$ способов, предполагаюших аналитическое продолжение. В частности, было отмечено, что способ регуляризации с помошью степенного множителя приводит при $d=3$ и $d=4$ к совершенно неприемлемым результатам, которые в случае однородных внешних полей противоречат обшепризнанным результатам более чем шестидесятилетней давности Гайзенберга и Ойлера [9] и Вайскопфа [10]. 
Поэтому в настоящей работе задача индуцирования вакуумной энергии сингулярным магнитным вихрем решена с использованием именно экспоненциальной регуляризации. В то же время показано, почему в данном случае к правильному результату приводит также и способ регуляризации, предполагаюший аналитическое продолжение по аргументу дзета-функции.

Благодарности. Работа выполнена при поддержке Государственного фонда фундаментальных исследований Министерства науки Украины (проект 2.4/320).

\section{Список литературы}

[1] А. И. Ахиезер, В. Б. Берестецкий. Квантовая электродинамика. М.: Наука, 1981.

[2] А.А. Гриб, С. Г. Мамаев, В. М. Мостепаненко. Квантовые эффекты в интенсивных внешних полях. М.: Атомиздат, 1980.

[3] Н. Бирелл, П. Девис. Квантованные поля в искривленном пространстве-времени. М.: Мир, 1984.

[4] Yu. A. Sitenko, A. Yu. Babansky. Mod. Phys. Lett. A. 1998. V. 13. P. 379.

[5] Ю.А. Ситенко, Д. Г. Ракитянский. ЯФ. 1998. Т. 61. С. 876.

[6] H. B. G. Casimir. Proc. K. Ned. Akad. Wetensc. 1948. V. 51. P. 793; Physica. 1953. V. 19. P. 846.

[7] E. Elizalde, S.D. Odintsov, A. Romeo, A.A. Bytsenko, C. Zerbini. Zeta Regularization Techniques with Applications. Singapore: World Scientific, 1994.

[8] Г. Бейтмен, А. Эрдейи. Высшие трансцендентные функции. Т.1. М.: Наука, 1973.

[9] W. Heisenberg, H. Euler. Z. Phys. 1936. V. 98. P. 714.

[10] V. Weisskopf. Kgl. Dansk. Vidensk. Selsk., Mat. -Fys. Medd. 1936. V. 16. № 6. P. 1.

Поступила в редакцию 8.VI.1998 г., после доработки 30.Х.1998 г. 